\title{
Three-Dimensional, Spin-Resolved Structure of Magnetic Vortex and Antivortex States in Patterned Co Films Using Scanning Ion Microscopy with Polarization Analysis
}

\author{
Jian Li and Carl Rau \\ Department of Physics and Astronomy, Rice Quantum Institute and Center for Nanoscience and Technology, Rice University, \\ Houston, Texas 77251, USA \\ (Received 11 February 2005; published 5 September 2006)
}

\begin{abstract}
Scanning ion microscopy with polarization analysis is utilized for three-dimensional spin mapping of the surface magnetization (SM) of circular Co dots created in situ by focused ion beam etching of $30 \mathrm{~nm}$ thin $\mathrm{Co} / \mathrm{Si}(100)$ films. From 3D scanning ion microscopy with polarization analysis spin maps, direct evidence is found for the existence of vortex-antivortex states with in-plane circular or hyperbolic SM components and a wide core with perpendicular SM components which oscillate in the outer region and become zero.
\end{abstract}

DOI: 10.1103/PhysRevLett.97.107201

PACS numbers: 75.60.Ch, 07.78.+s, 75.70.Rf

Recently, broad and intense scientific interest has focused on the physics of magnetism at the nanometer and micrometer scales. Studies of the surface magnetic structure (SMS) of small magnetic elements receive great attention. They allow the exploration of a wealth of new, fundamental SMSs that emerge when the thickness of the sample is of the order of or below the bulk Bloch wall thickness. Central to the fundamental understanding of these small magnetic elements is the precise knowledge of their three-dimensional (3D) SMSs and their dependence on the shape and size of the elements. Of further importance are the interactions between these small elements in patterned magnetic materials. This requires shapes of elements that exhibit minimized stray fields; for example, circular disks should possess flux-closure vortex states with only small external stray fields at remanence.

In recent years, the existence of $S, C$, flower, edgepinning, vortex, antivortex, and other so-called micromagnetic ground states has been predicted and to some extent directly or indirectly observed [1-16]. For many SMSs, however, their detailed 3D, spin-, and spatially resolved SMS is presently not yet known. Among these SMSs, fluxclosure magnetic vortex states, existing in circular magnetic elements, are paid great attention, because they are not only proposed for use in data storage [16], they are reported to possess some unique physical features, such as an overall in-plane circular SMS profile with a central nanometer-sized core possessing a perpendicular magnetization $[6,9,15]$, which plays an important role in the dynamics of microscopic magnets $[17,18]$.

Using magnetic force microscopy [6], Lorentz microscopy $[9,15]$, a combination of surface magneto-optical Kerr effect (SMOKE) hysteresis loops and micromagnetic simulations $[4,8]$, off-axis holography in transmission electron microscopy [10], and scanning electron microscopy with polarization analysis [13], the presence of magnetic vortices in patterned magnetic permalloy and Co elements was indirectly or directly verified. For Fe quantum dots, the existence of curling spin structures in vortex cores is also predicted from full-potential linear augmented-plane-wave calculations [19]. To the best of our knowledge, there is presently no fundamental information available about the detailed 3D spin structure of magnetic vortices which could help to obtain more insight into the fundamental physical properties of magnetic vortices.

In this Letter, we report the first experimental observation of the detailed 3D, spin-, and spatially resolved SMS of magnetic vortices and antivortices in circular (diameter $d=5-38 \mu \mathrm{m}$ ), thin (thickness $D=30 \mathrm{~nm}$ ), polycrystalline Co disks with a very small magnetocrystalline anisotropy where the competition between exchange and demagnetizing fields should predominantly be responsible for the basic SMS. From spin- and spatially resolved scanning ion microscopy with polarization analysis (SIMPA) spin maps, obtained at surfaces of circularshaped, patterned Co elements, after demagnetization of previously magnetized Co elements, we find that, depending on the diameter $d$ of the elements, the detailed SMS consists completely of single or multivortex states.

In SIMPA, a microfocused (spot size: $35 \mathrm{~nm}$ ) $25 \mathrm{keV}$ $\mathrm{Ga}^{+}$ion beam is scanned across a magnetic or nonmagnetic surface, causing the emission of spin-polarized or non-spin-polarized electrons $[20,21]$. The electrons emitted from the sample surface are collected by using an extracting lens system, and the electron spin polarization (ESP) is measured by using a Mott polarimeter. SIMPA offers some unique advantages compared to many other magnetic imaging techniques, because of its capability to produce vectorial maps of the SM by directly measuring the spatially resolved vector orientation and magnitude of the ESP, which is directly proportional to the magnitude and orientation of the SM of the local area probed by the focused ion beam [20,21]. The inelastic mean free path of the emitted electrons amounts to only a few monolayers [22], which explains the high surface sensitivity of SIMPA. 
We are analyzing the $3 \mathrm{D}$ components of the SM by detecting the 3D components of the ESP $\mathbf{P}$ of the emitted electrons. We note that only the $x$ and $y$ components can be measured simultaneously. The $z$ component is detected by measuring $P$ with the sample rotated by $45^{\circ}$. For details, we refer to the caption of Fig. 2. For the measurements, $30 \mathrm{~nm}$ thick, polycrystalline Co films are produced on $\mathrm{Si}(100)$ substrates by using electron beam evaporation at a rate of $0.03 \mathrm{~nm} / \mathrm{s}$ [23]. Circular-shaped magnetic elements $(d=5-30 \mu \mathrm{m})$ are fabricated by using a $1 \mathrm{nA}$ ion beam with a beam spot size of $100 \mathrm{~nm}$. Focused ion beam (FIB) etching is performed by circular (diameter $d^{\prime}$ ) etching starting at $d^{\prime}>3.5 \mathrm{~d}$ and ending at $d^{\prime}=d$.

The SMS for the Co elements is determined by using SIMPA at $0.08 \mathrm{nA}$ and a beam spot size of $35 \mathrm{~nm}$. From subsequently taken SIMPA images, no changes in the SMSs are observed [24]. For obtaining additional magnetic information, longitudinal SMOKE studies are performed.

A normalized SMOKE hysteresis loop obtained at the surface of a $30 \mathrm{~nm}$ thick, unpatterned $\mathrm{Co} / \mathrm{Si}(100)$ film is given in Fig. 1(a). Magnetic fields up to 160 Oe are applied. As shown in Fig. 1(a), a square magnetic hysteresis loop with a low coercivity field $H_{c}=14.8 \mathrm{Oe}(1,178 \mathrm{~A} / \mathrm{m})$ is found. No changes of size and shape of the square loop are observed upon rotation of the samples around the surface normal, which indicates the presence of zero or randomly oriented magnetocrystalline anisotropy due to the very good polycrystalline nature of the Co films. For electron beam evaporated $\mathrm{Co} / \mathrm{Si}$ films, similarly low coercive fields were found by Wu et al. [25] and Fernandez et al. [26]. In Ref. [26], it is pointed out that very small grain sizes cause a smoothing out of crystalline anisotropies leading to such small coercive fields. In order to quantify this, we calculate a reduced anisotropy constant $Q=K_{r} / K_{d}$, where $K_{r}$ is an appropriate residual anisotropy coefficient and $K_{d}$ is the stray field energy coefficient. For $K_{r}=H_{c} M_{s} / 2 \mu_{0}, K_{d}=$ $M_{s}{ }^{2} / 2 \mu_{0}$, and $M_{s}=1.4 \times 10^{6} \mathrm{~A} / \mathrm{m}$, we obtain $Q=$ $H_{c} / M_{s}=8.4 \times 10^{-4}$. We note that for epitaxial, fcc, or hcp Co thin films, $Q$ values are much higher $\left(Q_{\mathrm{fcc}}=\right.$

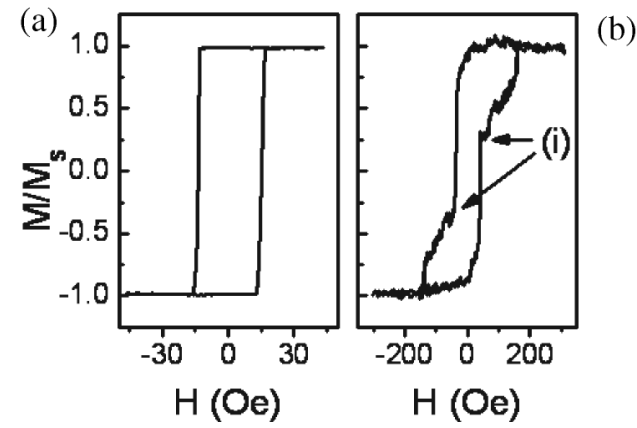

FIG. 1. (a) A normalized SMOKE hysteresis loop for a $30 \mathrm{~nm}$ ultrathin $\mathrm{Co} / \mathrm{Si}(100)$ film; (b) a normalized SMOKE hysteresis loop for a $d=6 \mu \mathrm{m}$ Co element created by circular FIB etching starting at $d^{\prime}=22 \mu \mathrm{m}$ and ending at $d^{\prime}=6 \mu \mathrm{m}$. $\left.5.25 \times 10^{-2} \quad[13,14], \quad Q_{\text {hcp }}=4.28 \times 10^{-1} \quad[27]\right)$. Figure 1(b) shows a SMOKE hysteresis loop obtained at the surface of a single Co element with $d=6 \mu \mathrm{m}$. Magnetic fields up to 500 Oe are applied. The SMOKE hysteresis loop has now an enhanced coercivity field $H_{c}=$ 38 Oe. From SIMPA measurements, it is found that vortex nucleation occurs at $H_{\mathrm{vn}} \approx 50$ Oe [see (i) in Fig. 1(b)]. Saturation magnetization, where vortex annihilation occurs, is reached at $H_{s} \approx 150 \mathrm{Oe}$.

Figure 2(a) shows a spin- and spatially resolved SIMPA spin map obtained at the surface of a FIB-created, circular $(d=6 \mu \mathrm{m})$, Co $(D=30 \mathrm{~nm}) / \mathrm{Si}(100)$ element. In order to obtain a clear plot of the $P$ vectors and to account for statistical fluctuations in electron count rates, for all spin maps, $P$ is averaged over four nearest neighboring points and then only every fourth $P$ vector is plotted, thereby reducing the density of $P$ vectors by a factor of 64. From the distribution of the in-plane ESP vectors $P_{\|}\left(P_{\|}^{2}=\right.$ $P_{x}{ }^{2}+P_{y}{ }^{2}$ ) (for details, see figure caption), it is clearly visible that the SMS is completely nonuniform and exhibits predominantly circular in-plane components. The curling of the $P_{\|}$vectors directly reveals the single vortex structure of the SM. No magnetic domains with uniform SM are found, which is due to the negligible magnetocrystalline anisotropy $\left(Q=8.4 \times 10^{-4}\right)$ of the Co elements.

From Fig. 2(a), it is visible that the magnitude of the inplane ESP $P_{\|}$decreases from the outer region with decreasing distance to the center of the vortex, where it becomes zero, indirectly indicating, with the assumption that the magnitude of the total ESP $\mathbf{P}$ is constant, the existence of an increasing out-of-plane component $P_{z}$ of the ESP, which, at the center of the vortex core, points perpendicularly out of the surface plane. This is more clearly visible from a horizontal SIMPA line scan through the center of the vortex. This is shown in the lower part of Fig. 2(b), which gives $P_{\|}$as a function of the $x$ coordinate from $x=-3 \mu \mathrm{m}$ to $x=+3 \mu \mathrm{m}$, where the center of the vortex is at $x=0$. At the center of the vortex, $P_{\|}=0$ is found, and, in the far outer region, $P_{\|}$amounts to $25 \%$. For the measurement of the perpendicular component $P_{z}$ of $\mathbf{P}$, a second SIMPA image is taken with the sample rotated $45^{\circ}$ around the $y$ axis. $P_{z}$ is obtained from $P_{x}{ }^{45}=\left(P_{x}-\right.$ $\left.P_{z}\right) \times 0.707$, with $P_{x}$ being the measured $x$ component of $\mathbf{P}$ before rotating and $P_{x}{ }^{45}$ the $x$ component of $\mathbf{P}$ measured at $45^{\circ}[20,28]$.

In the upper part of Fig. 2(b), for $P_{z}$, the results of a horizontal SIMPA line scan through the center of the vortex are given. At the center of the vortex, $P_{z}$ exhibits a maximum value $(25 \%)$ and decreases slowly towards zero, showing oscillations in the outer region $(|x|>$ $1.2 \mu \mathrm{m})$. These findings verify the existence of a vortex with a wide core $(|x|<1.2 \mu \mathrm{m})$ and an outer region $(1.2 \mu \mathrm{m}<|x|<3 \mu \mathrm{m})$ where $P_{z}$ oscillates and becomes zero. Note the reversed polarization in the outer region which compensates partially the positive polarization in 

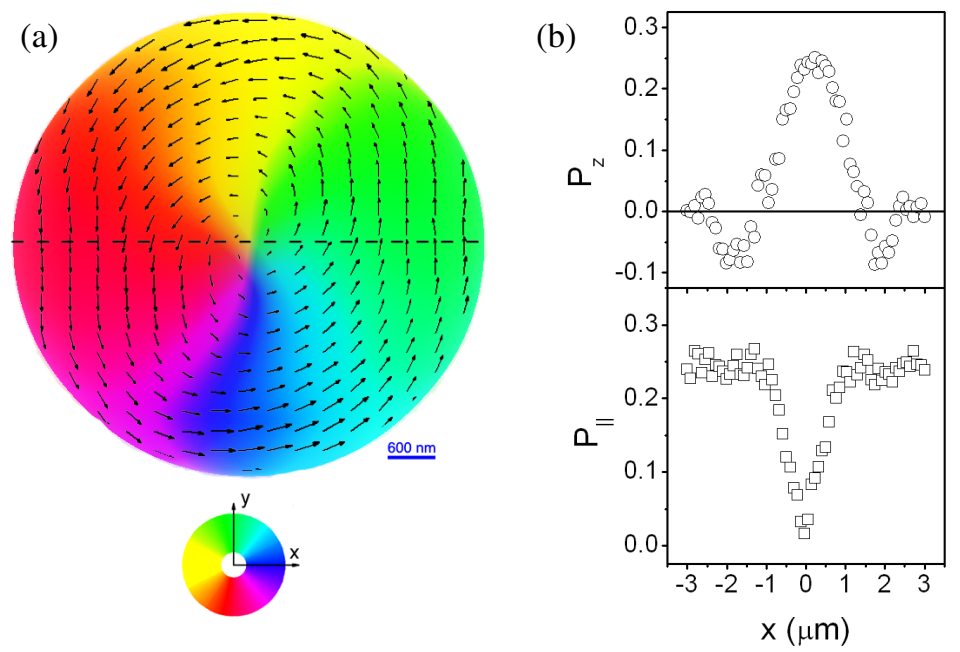

(c)

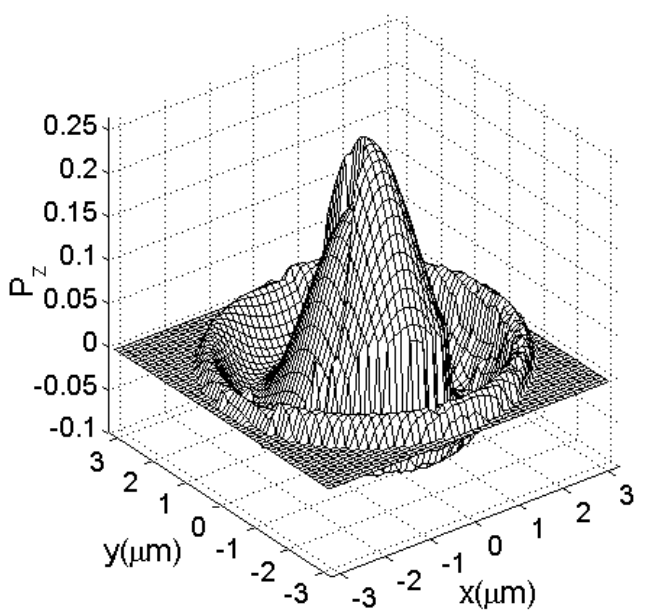

FIG. 2 (color online). (a) A SIMPA spin map of a $d=6 \mu \mathrm{m}$ Co element. The gray shades represent different orientations of $P_{\|}$. [See gray shade wheel in the lower part of (a) together with $x$ and $y$ axes; used for all figures]. The $\mathbf{z}$ axis is defined as $\mathbf{x} \times \mathbf{y}$. The local orientation and magnitude of the $P$ vectors is given by black arrows (used for all figures), the magnitude of $P$ being proportional to the length of the arrows. (b) Results of a horizontal SIMPA line scan [see dashed line in (a)] through the center of the vortex. The perpendicular SM component $P_{z}$ is shown in the upper part of the figure and the in-plane component $P_{\|}$is shown in the lower part of the figure. (c) A 3D plot of the $P_{z}$ components for the vortex shown in (a).

the core [1]. We remark that such oscillations have been predicted theoretically [29-31]. We calculate the magnitude $P$ of the total ESP $\mathbf{P}$ by using $P^{2}=P_{\|}{ }^{2}+P_{z}{ }^{2}$ for all surface points and find that $P$ is constant $(25 \%)$ across the surface of the Co element within experimental errors, which confirms our earlier assumption. This is also valid for the results presented in Fig. 2(b). The value of 25\% is consistent with earlier SIMPA measurements obtained at surfaces of $30 \mathrm{~nm}$ thin, continuous Co/Si films [21].

Using all measured $P_{z}$ line scans of the circular $(d=$ $6 \mu \mathrm{m}) \mathrm{Co} / \mathrm{Si}(100)$ element, in Fig. 2(c), a 3D plot of $P_{z}$ is shown. The wide core of the vortex with nonzero out-ofplane polarization components and the oscillations of $P_{z}$ in the outer region are clearly visible as well as significant and systematic deviations from a fully rotation-symmetric vortex structure, which are also noticeable in Fig. 2(a).

These findings directly reveal the importance of detecting all three components of the SM in order to obtain fundamental information on the SMS of magnetic vortices. We remark that the relative core widths (core width/disk diameter $d$ ) of approximately $30 \%$ found in our experiments seem to be well within the range of relative core widths $(10 \%-40 \%)$ reported in other experimental $(35 \%-$ $40 \%$ [32]) and theoretical (10\% [31], 20\% [6], 20\%-25\% $[29,30]$, and $25 \%$ [33]) studies for smaller elements.

In further SIMPA experiments, $d$ is varied between 5-38 $\mu \mathrm{m}$. For $5 \mu \mathrm{m}<d<13 \mu \mathrm{m}$, only single vortex states are observed. For $d>(13-15) \mu \mathrm{m}$, multivortex states including antivortex states are found. Figure 3(a) gives a SIMPA spin map of an FIB-created Co element for $d=18 \mu \mathrm{m}$. The SMS is completely nonuniform and exhibits three vortices and one antivortex (see SM profile within the white-dashed area). The three vortices possess a circular SM profile similar to that shown in Fig. 2(a) for $d=6 \mu \mathrm{m}$. Two vortices (v1 and $\mathrm{v} 3$ ) exhibit left-handed chirality and one vortex (v2) shows right-handed chirality with a circular SM profile. The antivortex (av), however, exhibits a hyperbolical SM profile [1]. As for the vortices, also for the antivortex, the magnitude of $P_{\|}$decreases slowly with decreasing distance to the center of the antivortex, pointing to the existence of nonzero out-of-plane components of the SMS of the antivortex.

From a SIMPA line scan along the white-dashed line shown in Fig. 3(a), we obtain $P_{z}(x)$, which is given in Fig. 3(b). The negative $P_{z}$ values measured for $|x|<3 \mu \mathrm{m}$ indicate the existence of the wide core of the antivortex (av) with negative, out-of-plane components of the SM. Between $-3 \mu \mathrm{m}>x>-9 \mu \mathrm{m}, P_{z}$ oscillates weakly $\left(P_{z}{ }^{\max }=+8 \%\right)$ and becomes zero. For $3 \mu \mathrm{m}<x<$ $9 \mu \mathrm{m}, P_{z}$ increases to $+25 \%$ at $x \approx 6.5 \mu \mathrm{m}$ and decreases to zero towards $x=9 \mu \mathrm{m}$. This region seems to be a superposition of the wide core and the outer region of the vortex (v2) with the weakly oscillating $P_{z}$ component of the antivortex in the outer region $(3 \mu \mathrm{m}<|x|<9 \mu \mathrm{m})$ of the antivortex.

In conclusion, it is shown that SIMPA provides an excellent means to study the detailed spin- and spatially resolved 3D SMS of nonuniform (curling) micromagnetic states, such as magnetic vortices and antivortices, in patterned magnetic films. The magnetic vortex-antivortex states possess a circular or hyperbolic SMS, respectively, with a central vortex-antivortex core with nonzero perpendicular SM components and wide extended tails with nonzero out-of-plane components of the SM due to the small 

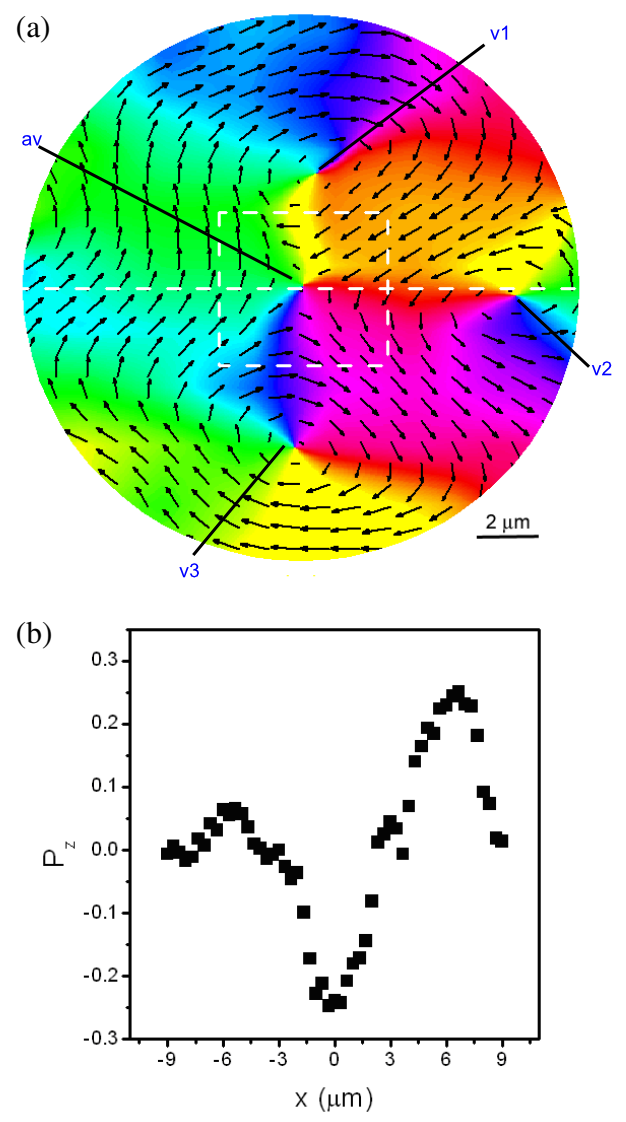

FIG. 3 (color online). (a) A SIMPA spin map obtained at the surface of a FIB-created, circular $d=18 \mu \mathrm{m}$ Co element. (b) Results for $P_{z}$ obtained from of a horizontal SIMPA line scan along the white-dashed line shown in (a).

reduced anisotropy constant $Q$. It is further observed that the vortex core width as well as the relative core width (core width/disk diameter) decrease with decreasing diameter $d$ of the disks. We believe that this most recent advancement of SIMPA to detect all three components of the SM will help to obtain a coherent physical picture of magnetic vortices and other sophisticated SMSs. The results show that 3D SIMPA spin mapping can develop into an important and efficient tool to find desired, optimized micromagnetic ground states for patterned magnetic systems to be used for chirality-based devices.

[1] A. Hubert and R. Schäfer, Magnetic Domains: The Analysis of Magnetic Microstructures (Springer-Verlag, Berlin, 1998).
[2] Y. Zheng and J.-G. Zhu, J. Appl. Phys. 81, 5471 (1997).

[3] W. Rave and A. Hubert, IEEE Trans. Magn. 36, 3886 (2000).

[4] J. Shi and S. Tehrani, Appl. Phys. Lett. 77, 1692 (2000).

[5] M.E. Schabes and H. N. Bertram, J. Appl. Phys. 64, 1347 (1988).

[6] T. Shinjo et al., Science 289, 930 (2000).

[7] J. Miltat and A. Thiaville, Science 298, 555 (2002).

[8] R.P. Cowburn, D. K. Koltsov, A. O. Adeyeye, M.E. Welland, and D. M. Tricker, Phys. Rev. Lett. 83, 1042 (1999).

[9] J. Raabe et al., J. Appl. Phys. 88, 4437 (2000).

[10] R. E. Dunin-Borkowski et al., Appl. Phys. Lett. 75, 2641 (1999).

[11] S. P. Li et al., Phys. Rev. Lett. 86, 1102 (2001).

[12] J. Rothman et al., Phys. Rev. Lett. 86, 1098 (2001).

[13] C. A. F. Vaz, L. Lopez-Diaz, M. Kläui, J. A. C. Bland, T. L. Monchesky, J. Unguris, and Z. Cui, Phys. Rev. B 67, 140405(R) (2003).

[14] M. Kläui et al., Phys. Rev. B 68, 134426 (2003).

[15] M. Schneider, H. Hoffmann, and J. Zweck, Appl. Phys. Lett. 77, 2909 (2000); 79, 3113 (2001).

[16] J.-G. Zhu, Y. Zheng, and G. A. Prinz, J. Appl. Phys. 87, 6668 (2000).

[17] S. B. Choe et al., Science 304, 420 (2004).

[18] V. Novosad et al., Phys. Rev. B 72, 024455 (2005).

[19] A. J. Freeman, K. Nakamura, and T. Ito, J. Magn. Magn. Mater. 272, 1122 (2004).

[20] N. J. Zheng and C. Rau, Mater. Res. Soc. Symp. Proc. 313, 723 (1993).

[21] J. Li and C. Rau, J. Appl. Phys. 95, 6527 (2004).

[22] D. L. Abraham and H. Hopster, Phys. Rev. Lett. 58, 1352 (1987).

[23] W. C. Uhlig et al., J. Appl. Phys. 91, 6943 (2002).

[24] J. Li and C. Rau, Nucl. Instrum. Methods Phys. Res., Sect. B 230, 518 (2005).

[25] Y. Wu, J. Tsay, Sh. Chen, T. Fu, and Ch. Shern, Jpn. J. Appl. Phys. 40, 6825 (2001).

[26] A. Fernandez, M. R. Gibbons, M. A. Wall, and C.J. Cerjan, J. Magn. Magn. Mater. 190, 71 (1998).

[27] M. Grimsditch, E. E. Fullerton, and R. L. Stamps, Phys. Rev. B 56, 2617 (1997).

[28] M. Hoesch et al., J. Electron Spectrosc. Relat. Phenom. 124, 263 (2002).

[29] W. Scholz et al., J. Magn. Magn. Mater. 266, 155 (2003).

[30] K. Yu. Guslienko, W. Scholz, R.W. Chantrell, and V. Novosad, Phys. Rev. B 71, 144407 (2005).

[31] J. K. Ha, R. Hertel, and J. Kirschner, Phys. Rev. B 67, 224432 (2003).

[32] J. M. García-Martín, A. Thiaville, J. Miltat, T. Okuno, L. Vila, and I. Piraux, J. Phys. D: Appl. Phys. 37, 965 (2004).

[33] K. L. Metlov and K. Yu. Guslienko, J. Magn. Magn. Mater. 242-245, 1015 (2002). 